\title{
Physiotherapy, and speech and language therapy intervention for patients with refractory chronic cough: a multicentre randomised control trial
}

\author{
Sarah A F Chamberlain Mitchell, ${ }^{1,2}$ Rachel Garrod, ${ }^{3}$ Lynne Clark, ${ }_{1}^{4}$ Abdel Douiri, ${ }^{5,6}$ \\ Sean M Parker, ${ }_{1}^{7}$ Jenny Ellis, ${ }^{7}$ Stephen J Fowler, ${ }^{8}$ Siobhan Ludlow, ${ }_{1}{ }^{3}$ James H Hull, ${ }^{10}$ \\ Kian Fan Chung, ${ }^{10}$ Kai K Lee, ${ }^{1}$ H Bellas, ${ }^{11}$ Anand Pandyan, ${ }^{2}$ Surinder S Birring ${ }^{1}$
}

\begin{abstract}
- Additional material is published online only. To view please visit the journal online (http://dx.doi.org/10.1136/ thoraxjn-2016-208843).
\end{abstract}

For numbered affiliations see end of article.

\section{Correspondence to Dr Sarah Ann Frances Chamberlain Mitchell, Division of Asthma, Allergy and Lung Biology, King's College London, Denmark Hill, London SE9 5RS, UK: s.chamberlain.mitchell@ keele.ac.uk}

Received 29 April 2016 Revised 3 August 2016 Accepted 19 August 2016 Published Online First 28 September 2016

\section{SLinked}

- http://dx.doi.org/10.1136/ thoraxjnl-2016-209484

\section{CrossMark}

\author{
To cite: Chamberlain \\ Mitchell SAF, Garrod R, \\ Clark L, et al. Thorax
} 2017;72:129-136.

\section{ABSTRACT}

Background Physiotherapy, and speech and language therapy are emerging non-pharmacological treatments for refractory chronic cough. We aimed to investigate the efficacy of a physiotherapy, and speech and language therapy intervention (PSALTI) to improve health-related quality of life (HRQoL) and to reduce cough frequency in patients with refractory chronic cough.

Methods In this multicentre randomised controlled trial, patients with refractory chronic cough were randomised to four weekly 1:1 sessions of either PSALTI consisting of education, laryngeal hygiene and hydration, cough suppression techniques, breathing exercises and psychoeducational counselling or control intervention consisting of healthy lifestyle advice. We assessed the change in HRQoL at week 4 with the Leicester Cough Questionnaire (LCQ). Secondary efficacy outcomes included 24-hour objective cough frequency (Leicester Cough Monitor) and cough reflex sensitivity. The primary analysis used an analysis of covariance adjusted for baseline measurements with the intention-to-treat population. This study was registered at UK Clinical Research Network (UKCRN ID 10678).

Findings Between December 2011 and April 2014, we randomly assigned 75 participants who underwent baseline assessment (34 PSALTI and 41 controls). In the observed case analysis, HRQoL (LCQ) improved on average by $1.53(95 \% \mathrm{Cl} 0.21$ to 2.85$)$ points more in PSALTI group than with control $(p=0.024)$. Cough frequency decreased by $41 \%(95 \% \mathrm{Cl} 36 \%$ to $95 \%)$ in PSALTI group relative to control $(p=0.030)$. The improvements within the PSALTI group were sustained up to 3 months. There was no significant difference between groups in the concentration of capsaicin causing five or more coughs.

Interpretation Greater improvements in HRQoL and cough frequency were observed with PSALTI intervention. Our findings support the use of PSALTI for patients with refractory chronic cough.

Trial registration number UKCRN ID 10678 and ISRCTN 73039760; Results.

\section{INTRODUCTION}

Chronic cough, defined as a cough lasting more than 8 weeks, ${ }^{1}$ is a prevalent disorder in both the community $^{2}$ and secondary care sectors, accounting for up to $20 \%$ of respiratory outpatient clinic referrals. ${ }^{13}$

\section{Key messages}

What is the key question?

- Is physiotherapy, and speech and language therapy intervention (PSALTI) effective for patients with refractory chronic cough?

What is the bottom line?

- PSALTI significantly reduced objective cough frequency and significantly improved health related quality of life when compared with control intervention.

\section{Why read on?}

- This study is the first multicentred randomised controlled trial that demonstrates improvements with PSALTI compared with control intervention using objective outcome measures.

The most common causes of cough in a nonsmoking patient with a normal chest radiograph and spirometry are asthma, gastro-oesophageal reflux disease and rhinitis (upper airway cough syndrome). ${ }^{145}$ For a significant number of patients, the cough may remain unexplained or refractory to treatment despite extensive investigation and therapeutic trials. ${ }^{6}$ Cough is associated with significant physical and psychological morbidity as well as impaired quality of life. ${ }^{7-9}$ There are a few effective antitussive therapies for refractory chronic cough. ${ }^{10}{ }^{11}$ Recent studies suggest a potential role for gabapentin, pregabalin, amitriptyline, morphine and $\mathrm{P} 2 \mathrm{X} 3$ receptor inhibitors, but they are all associated with significant side effects. ${ }^{12-16}$

Non-pharmacological therapies for refractory chronic cough have shown promising results in a few studies and no significant adverse effects. ${ }^{17}$ Non-pharmacological therapies are generally delivered by physiotherapists or speech and language therapists, and key components include education, cough suppression techniques, including breathing exercises, vocal hygiene and hydration, and psychoeducational counselling. ${ }^{15}{ }^{17-23}$ Vertigan et al ${ }^{19}$ conducted the only randomised controlled trial of a non-pharmacological intervention for refractory chronic cough and found significantly greater 
improvements in symptoms of cough for speech pathology management compared with control (general healthy lifestyle advice). The benefits of speech pathology management on objectively measured cough frequency, cough reflex sensitivity and health-related quality of life (HRQoL) have not been assessed in a controlled clinical trial, limiting the generalisability of the findings. The minimal clinically important difference of the cough symptom score used in this study has not been defined. Furthermore, the longer term effect of therapy is not known. ${ }^{17} \mathrm{~A}$ recent study by Patel et al ${ }^{20}$ investigated coughsuppression physiotherapy for refractory chronic cough in 23 participants and found a significant improvement in coughrelated quality of life, but this study also did not include a control intervention.

This study therefore aimed to assess the effect of an intervention using both physiotherapy and speech and language therapy techniques (physiotherapy, and speech and language therapy intervention, PSALTI) on HRQoL, objective cough frequency, cough reflex sensitivity and cough severity using a randomised controlled design.

\section{METHODS}

A multicentre, single-blinded randomised controlled trial was conducted across three hospitals in the UK (King's College Hospital NHS Foundation Trust, Lancashire Teaching Hospitals NHS Foundation Trust and Northumbria Healthcare NHS Foundation Trust). Two further sites, Royal Brompton \& Harefield NHS Foundation Trust and Guy's and St Thomas' NHS Foundation Trust) were recruitment-only sites, and participants were referred to King's College Hospital to receive the intervention. The study was undertaken between December 2011 and April 2014.

\section{Participants and randomisation}

Eligible participants were identified as adults with chronic cough (defined as duration $>2$ months), with normal chest $\mathrm{X}$-ray, minimal sputum production $(<10 \mathrm{~mL}$ sputum a day) and who had negative investigations and/or failed treatment trials for asthma, gastro-oesophageal reflux disease and rhinitis, as per British Thoracic Society guidelines. ${ }^{1}$ Participants were excluded if they had an upper respiratory tract infection in the past four weeks, were taking ACE inhibitors medication, were current smokers or had a known respiratory disease (such as lung cancer, pneumonia, pulmonary fibrosis, sarcoidosis, pleural effusion, bronchiectasis). Participants were also excluded if they had vocal cord nodules, malignancy or evidence of active aspiration.

Once participants had given written consent and completed baseline assessments, they were registered into the randomisation service provided by the King's Clinical Trials Unit, King's College London. This prevented foreknowledge of treatment assignment for the study researchers. Group allocation was concealed from participants until they had completed the study and all postintervention assessments. Participants were block-randomised, stratified by age (above and below 50 years old) and gender.

\section{Control intervention}

Participants attended weekly sessions and received one-to-one standardised healthy lifestyle advice from a healthcare professional (nurse, physiotherapist or speech and language therapist) over 4 weeks. The control intervention was based on that used in the trial reported by Vertigan et al. ${ }^{19}$ The initial session covered general advice on exercise and physical activity, second session dietary and nutritional advice, third session stress management and fourth session relaxation. The material covered in each session was based on healthy lifestyle advised by the UK Department of Health and National Health Service. ${ }^{24-26}$ The sessions were standardised for all sites by using the same written prompts for therapists and educational information. Face-to-face training was provided for all site therapists who delivered the healthy lifestyle intervention. The duration of all trial sessions was $45 \mathrm{~min}$, except the initial session which was 1 hour.

\section{PSALTI intervention}

Participants attended weekly sessions and received one-to-one treatment from a healthcare professional (physiotherapist or speech and language therapist) over 4 weeks. Session durations were the same as for the control group. The intervention was based on previous speech pathology management and cough suppression physiotherapy studies for refractory chronic cough reported by Vertigan et $a l^{19}$ and Patel et al, ${ }^{20}$ respectively (table 1). The first session focused on educating participants about chronic cough, introduction to laryngeal hygiene and hydration techniques and cough suppression/distraction techniques. The second and third sessions covered cough suppression techniques in more detail, including breathing exercises (table 1). Nasal douching or steam inhalations were recommended to participants with nasal congestion. In the third session, psychoeducational counselling techniques were covered with the aid of an information booklet developed jointly by the main study researcher and clinical psychologist at the primary research site. The fourth session consisted of reinforcing all aspects of PSALTI. All components of PSALTI were delivered; however, the focus and emphasis on individual techniques varied for each participant, determined by the treating therapist. An Airway clearance technique (Active cycle of breathing technique, ACBT) was included in the PSALTI treatment if the participant's sputum production was close to the upper limit of sputum exclusion criteria. The standardisation of treatment between different hospitals was increased by the use of written treatment plans and educational material. All therapists delivering the

Table 1 PSALTI components

\begin{tabular}{ll}
\hline PSALTI component & Technique \\
\hline Education & $\begin{array}{l}\text { Educate patients on the cough reflex, chronic cough } \\
\text { and cough reflex hypersensitivity. } \\
\text { Explain the negative effects of repeated coughing. } \\
\text { Educate patients on voluntary control of cough. }\end{array}$ \\
$\begin{array}{l}\text { Increase frequency and volume of water and } \\
\text { noryngeal hygiene and }\end{array}$ & $\begin{array}{l}\text { Reduce caffeine and alcohol intake. } \\
\text { Promote nasal breathing. }\end{array}$ \\
Cough control & $\begin{array}{l}\text { Teach patients to identify their cough triggers. } \\
\text { Teach patients to use cough suppression or } \\
\text { distraction techniques at the first sign or sensation } \\
\text { of the need or urge to cough. These } \\
\text { cough-suppression/distraction techniques include: } \\
\text { forced swallowing, sipping water and sucking } \\
\text { sweets. } \\
\text { Teach patients breathing exercises: breathing pattern } \\
\text { re-education promoting relaxed abdominal breathing } \\
\text { pattern technique; pursed lip breathing to use to } \\
\text { control cough. } \\
\text { Motivate patients, reiterate the techniques and the } \\
\text { aims of therapy. } \\
\text { Behaviour modification: to try to reduce } \\
\text { over-awareness of the need to cough. } \\
\text { Stress and anxiety management }\end{array}$ \\
Psychoeducational &
\end{tabular}

Chamberlain Mitchell SAF, et al. Thorax 2017;72:129-136. doi:10.1136/thoraxjnl-2016-208843 
treatment were trained in PSALTI prior to commencing the study by the main study researcher.

\section{Primary efficacy endpoint}

HRQoL was assessed with the Leicester Cough Questionnaire (LCQ) at week 4, the primary endpoint. ${ }^{8}$ The LCQ is a validated 19-item cough-specific health-related quality of life questionnaire. Overall scores range from 3 to 21, with a higher score indicating a better HRQoL. The minimal important difference for LCQ is 1.3. ${ }^{27}$ Participants independently completed questionnaires at baseline, at 4 weeks (after fourth treatment session) and at 3-month follow-up. Questionnaires were then placed in sealed envelopes to avoid influencing the treating therapist.

\section{Secondary efficacy endpoints}

Secondary endpoints were assessed at baseline, 4 weeks and 3 months. Objective cough frequency was assessed with the Leicester Cough Monitor (LCM), a validated, objective, automated and ambulatory cough monitoring device. ${ }^{28}$ The LCM consists of an MP3 recording device (Phillips 662 MP3 recorder, UK), external microphone and automated cough detection software. The LCM has been used in previous clinical trials of gabapentin and erythromycin. ${ }^{12} 29$ Participants wore the device for 24 hours at baseline, at 4 weeks (after fourth treatment session) and 3-month follow-up and were instructed to resume their normal daily activities during this time period. The number of coughs per hour $\left(\mathrm{CF}_{\text {perhour }}\right)$ was recorded.

Capsaicin cough challenge was assessed in a subset of the participants (Kings College Hospital Foundation Trust and Northumbria Healthcare NHS Foundation Trust) to measure participants' cough reflex sensitivity at baseline and at 4 weeks (after fourth treatment session). Doubling concentrations of capsaicin solution ranging from 0 (saline), 0.49 to $1000 \mu \mathrm{m}$ were administered as per European Respiratory Society guidelines. ${ }^{30}$ A doseresponse capsaicin cough testing method was used. ${ }^{30}$ The nebuliser output was set to $0.01 \mathrm{~mL} /$ breath. The test was discontinued when five or more coughs were induced (C5). In addition, the dose that induced two or more coughs (C2) was recorded.

Cough severity in the past 2 weeks was assessed by a visual analogue scale (VAS; $0-100 \mathrm{~mm}$ ) as per American College of Chest Physicians guidelines. ${ }^{31}$ The vocal performance questionnaire (VPQ), ${ }^{32}$ a 12 -item tool was used to assess participants' perceived impact on their voice, since a high prevalence of voice disorders in patients with chronic cough has been reported. ${ }^{33}$ A score $>12$ indicates dysphonia. ${ }^{32}$ General health and mood were assessed by Short Form 36 (SF-36) and Hospital Anxiety and Depression Scale (HADS). ${ }^{34}{ }^{35}$ HADS is a 14-item questionnaire, a score for either subscale $\geq 8$ indicates mild symptoms, $\geq 11$ moderate and $\geq 15$ severe. SF-36 generates two summary scores, physical component summary score (PCS) and mental component summary score; both range from zero to hundred, and a higher score indicates better self-reported health. ${ }^{36}$

\section{Ethics and trial registration}

All protocols were approved by the London-Chelsea National Research Ethics Service (NRES) Committee (11-LO-0504). All participants provided written informed consent, and the study was registered with the UK Clinical Research Network (UKCRN ID 10678) and ISRCTN (73039760).

\section{Role of funding source}

The funding bodies had no role in study design, collection, analysis and interpretation of data, in the writing of the report or in the decision to submit for publication.

\section{STATISTICAL ANALYSIS AND SAMPLE SIZE}

Power calculations for the primary outcome (LCQ score) were performed based on estimates from a previous study, ${ }^{37}$ reporting a mean LCQ score in patients with chronic cough of 14.03 (SD: 3.87). Group sample sizes of 33 in each group achieve $80 \%$ power with a significance level of 5\% to detect a LCQ change of 2.7 (seen in our pilot study). Allowing for a 25\% dropout, we aimed to recruit 88 patients in total.

For each of the variables analysed, univariate descriptive statistics were summarised by randomised group to provide an overview of the data. Summary measures for the baseline characteristics of each group were presented as mean and SD for continuous 'approximate' normally distributed variables, medians and IQRs for nonnormally distributed variables, and frequencies and percentages for categorical variables. Univariate analyses were performed to compare study group using appropriate statistical tests according to the type and the distribution of the data: independent t-test or Mann-Whitney for continuous variables. Cough frequency and capsaicin data were log-transformed prior to analysis.

Primary efficacy analysis, change in LCQ at week 4, was based on analysis of covariance (ANCOVA) adjusted for the baseline LCQ measurements. The ANCOVA analysis was repeated to adjust for centre and specialty of the treating therapist. The analysis used data from the intention-to-treat (ITT) basis population, which included all randomised participants who had received at least one treatment session. In this analysis, only observed data were included, and no imputation was used for missing data. We also performed an analysis on a per-protocol population which included participants who completed end-of-treatment (week 4) cough assessments and who did not deviate from the protocol (established before unmasking). Sensitivity analyses were performed for missing data according to different predefined populations using ANCOVA, with multiple imputations (see online supplementary appendix methods and online supplementary appendix - table 1). ${ }^{38}$ Similar sensitivity analyses were also performed for objective log-transformed cough frequency endpoints (see online supplementary appendix methods and online supplementary appendix - table 2).

The secondary efficacy analysis used data from the ITT population. In these analyses, ANCOVA was used adjusting for baseline variables, and only observed data were included without imputation for missing data. A value of $\mathrm{p}<0.05$ was considered statistically significant. All analyses were made using STATA V.12 software (StataCorp LP, College Station, Texas, USA).

\section{RESULTS}

\section{Participants}

Seventy-five participants were randomised and had baseline assessments. One additional participant was randomised to the PSALTI group but did not attend baseline assessments. Four participants did not receive any treatment (PSALTI group $(n=3)$ : myocardial infarction prior to treatment, unable to travel to hospital and insufficient time for the study; control group $(n=1)$ : undisclosed illness prior to start of treatment). The ITT population for LCQ primary analysis consisted of 71 participants (figure 1, and see online supplementary appendix methods and online supplementary appendix - table 1). A total of four participants in the control group and eight participants in the PSALTI group did not receive or complete all treatments for reasons stated in figure 1. Forty-nine participants completed 3-month follow-up. The consort study flow is described in figure 1 . The baseline characteristics of the randomised participants are described in table 2 . The groups were well matched, with the exception of SF-36 PCSs (higher in the control group). 


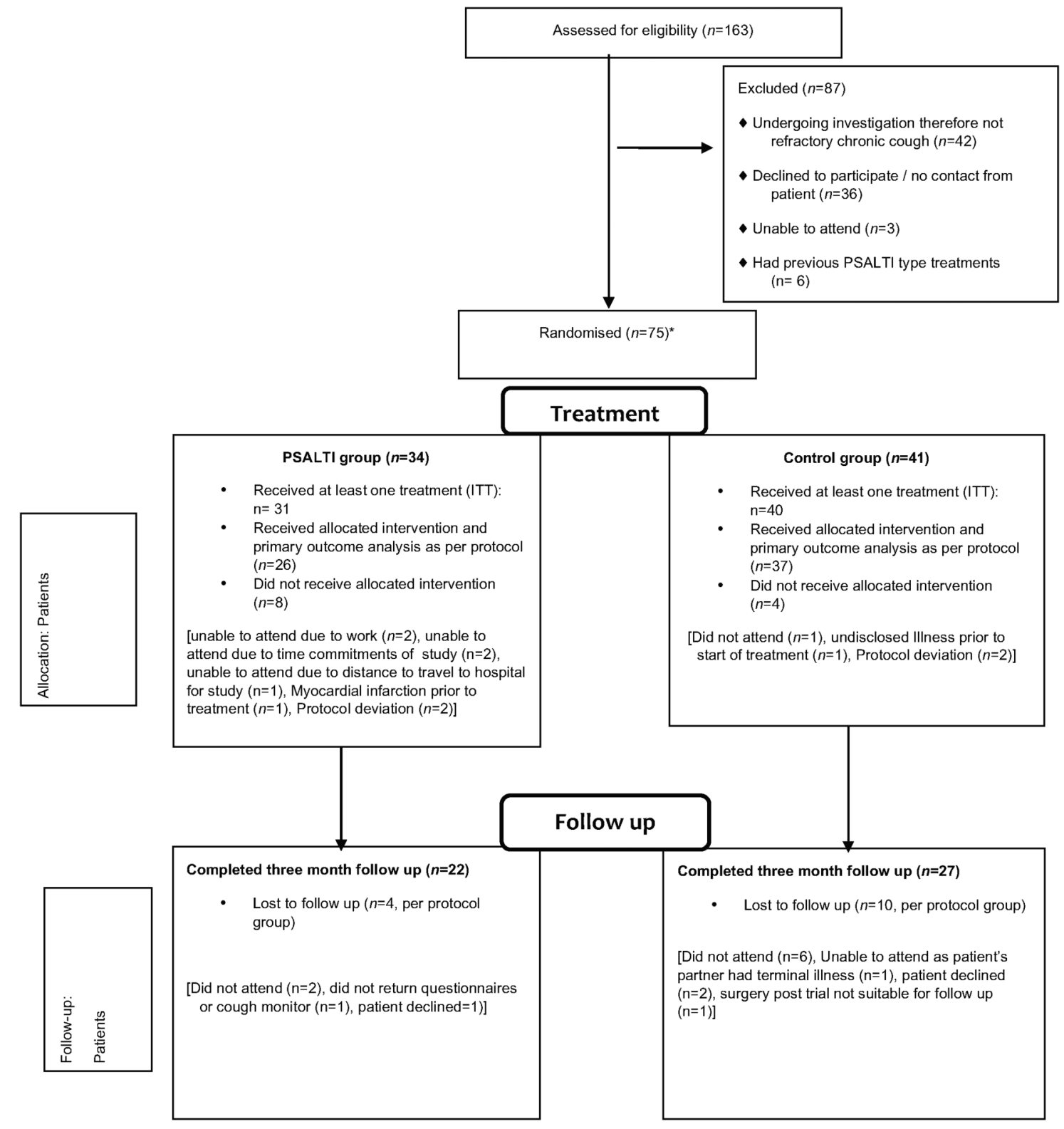

*One additional participant was randomised and withdrew before baseline assessments.

Figure 1 Trial CONSORT flow diagram.

\section{HRQOL—primary efficacy endpoint}

There was an improvement in the mean total LCQ score at 4 weeks with PSALTI; baseline 10.4 vs 14.4 at 4 weeks, mean difference 3.4, $\mathrm{p}<0.001$. This improvement was larger than that in the control group; baseline mean 11.9 vs 13.4 at 4 weeks, mean difference 1.66, $\mathrm{p}<0.001$ (see online supplementary appendix-table 3). Total LCQ score at 4 weeks improved by a mean 1.53 (95\% CI 0.21 to 2.85 ) units more in the PSALTI group than that in control $(p=0.024)$, table 3 . When adjusted for centre and specialty of therapist, the LCQ score at 4 weeks improved by a mean of 1.53 (95\% CI 0.20 to 2.86), $\mathrm{p}=0.024$. The improvement in LCQ with PSALTI was consistent in the per-protocol and sensitivity analyses (see online supplementary appendix-table 1). The LCQ improvement was sustained from week 4 to 3 months for both groups, but there was no significant difference between groups at 3 months (table 3). The LCQ scores and within-group differences are presented in online supplementary appendix - table 3 and table 4 in main article respectively. Primary outcome LCQ data (baseline or week 4) were missing in $6.7 \%$ of participants. There were no adverse or serious adverse events reported for both interventions.

\section{Objective cough frequency}

There was a greater reduction in cough frequency after 4 weeks of treatment in the PSALTI group; geometric mean (SD) 17.0 (2.4) to 9.0 (3.3) coughs per hour $(\mathrm{p}=0.002)$ vs $17.0(2.3)$ to 16.0 (2.2) coughs per hour after control $(p=0.205)$ (table 4). The control-adjusted decrease in cough frequency per hour in PSALTI was $41 \%$ (95\% CI ( $36 \%$ to $95 \%), p=0.030$, ANCOVA) at 4 weeks in the primary ITT analysis (table 3 ). This reduction was also sustained at 3 months (figure 2). The reduction in cough frequency with PSALTI was consistent in per-protocol and sensitivity analyses (see online supplementary appendixtable 2). 


\section{Other questionnaire data}

There were no significant between-group differences for change (week 4 minus baseline) in VPQ, depression, anxiety or SF-36 (table 3). There was a greater reduction in VAS cough severity in the PSALTI group compared with control $(\mathrm{p}=0.084$, table 3$)$. Cough severity VAS within both groups between week 4 and baseline; was significantly reduced control: $p=0.007$, PSALTI $\mathrm{p}<0.001$ (table 4).

Table 2 Baseline demographic and clinical characteristics of randomised study participants

\begin{tabular}{|c|c|c|c|}
\hline Characteristic & Control $(n=41)$ & PSALTI $(n=34)$ & p Value \\
\hline Age (years) & $56(48-67)$ & $61(53-67)$ & 0.239 \\
\hline Female, n (\%) & $26(63)$ & $25(71)$ & 0.459 \\
\hline Cough duration (months) & $48(24-126)$ & $60(30-126)$ & 0.279 \\
\hline $\mathrm{FEV}_{1}(\mathrm{~L}$, observed), mean (SD) & $2.7(0.9)$ & $2.6(0.7)$ & 0.517 \\
\hline $\mathrm{FEV}_{1} / \mathrm{FVC}(\%)$, mean (SD) & $76(8.2)$ & $76(5.0)$ & 0.686 \\
\hline LCQ, mean(SD) & $11.9(3.5)$ & $10.4(3.6)$ & 0.073 \\
\hline Cough severity VAS & $65(40-83)$ & $63(49-75)$ & 0.652 \\
\hline SF-36 PCS, & $47.1(41.7-53.6)$ & $41.1(35.6-49.1)$ & $0.033^{*}$ \\
\hline SF-36 MCS & $47.7(38.3-54.9)$ & $49.9(40.5-57.0)$ & 0.763 \\
\hline HADS-Anxiety & $7(3-10)$ & $7(4-10)$ & 0.785 \\
\hline HADS-Depression & $4(1-8)$ & $5(2-6)$ & 0.620 \\
\hline VPQ & $17(11-22)$ & $21(13-27)$ & 0.158 \\
\hline $\mathrm{CF}_{\text {per hour }} \dagger$ & $17.0(0.4)$ & $17.0(0.4)$ & 0.983 \\
\hline$C 2(\mu \mathrm{m}) \dagger$ & $4.01(0.69)$ & $4.740 .62)$ & 0.677 \\
\hline $\mathrm{C} 5(\mu \mathrm{m}) \dagger$ & $9.33(0.56)$ & $8.25(0.51)$ & 0.708 \\
\hline \multicolumn{4}{|c|}{$\begin{array}{l}\text { Data presented as median (IQR) unless otherwise stated. } \\
{ }^{*} \mathrm{p}<0.05 \text {. } \\
+\mathrm{tGeometric} \text { mean (log SD). } \\
\mathrm{C} \text {, capsaicin cough challenge-concentration that resulted in two or more coughs; } \\
\mathrm{C} \text {, capsaicin cough challenge-concentration that resulted in five or more coughs; } \\
\mathrm{CF} \text { per hour, cough frequency per hour over a 24-hour period; HADS, Hospital Anxiety } \\
\text { and Depression Scale; LCQ, Leicester Cough Questionnaire; MCS, mental component } \\
\text { score; PCS, physical component score; PSALTI, physiotherapy, and speech and } \\
\text { language therapy intervention; SF-36, short form } 36 \text { questionnaire; VAS, visual } \\
\text { analogue scale; VPQ, vocal performance questionnaire. }\end{array}$} \\
\hline
\end{tabular}

\section{Cough reflex sensitivity}

Sixty participants $(80 \%$ of ITT group) underwent capsaicin cough challenge. No significant differences between groups were observed for C2 $(\mathrm{p}=0.575)$ or C5 $(\mathrm{p}=0.512)$ (table 3$)$. There was a within-group reduction in C5 with PSALTI $(p=0.035)$, but not with control $(p=0.469)$ (table 4$)$.

\section{DISCUSSION}

This study evaluated the efficacy of a physiotherapy, and speech and language therapy intervention for patients with refractory chronic cough in a randomised controlled trial. There was a clinically and statistically significant improvement in health-related quality of life with PSALTI compared with control intervention. This was supported by a significant reduction in cough frequency measured objectively. The improvement in health-related quality of life was sustained at a 3-month follow-up visit. There was no significant change in cough reflex sensitivity between groups.

Our findings represent an advance from those reported in an earlier study by Vertigan et al. ${ }^{19}$ Our study is the first multicentre trial of non-pharmacological treatment reported in chronic cough and has the potential to provide the evidence base for access to therapy. Vertigan $e t a l^{19}$ reported a statistically significant reduction in cough symptoms scores, but did not include HRQoL or objective assessment with cough frequency monitors. In contrast, we assessed HRQoL, objective cough frequency, cough severity VAS and cough reflex sensitivity. We were also able to demonstrate both a clinically and statistically significant improvement in our primary endpoint, because the minimally important difference (MID) of the LCQ has been defined. ${ }^{27}$ We have shown that the benefits of PSALTI are sustained after discontinuation of therapy, in contrast to Vertigan et $a l^{19}$ who did not report follow-up data for their participants. One of the strengths of our study was the involvement of multiple centres, the use of standardised treatment protocols and the inclusion of both physiotherapists and speech and language therapists delivering the treatment.

HRQoL, as assessed with the LCQ, was selected as the primary outcome measure because it is perhaps the most

Table 3 Primary and secondary efficacy endpoint analysis: change between PSALTI and control groups at baseline to 4 weeks and at 4 weeks to 3 month follow-up

\begin{tabular}{|c|c|c|c|c|}
\hline & \multicolumn{2}{|l|}{$\begin{array}{l}\text { Between-group difference } \\
\text { Baseline to } 4 \text { weeks }\end{array}$} & \multicolumn{2}{|c|}{$\begin{array}{l}\text { Between-group difference } \\
4 \text { weeks to 3-month follow-up }\end{array}$} \\
\hline & Mean difference $(95 \% \mathrm{Cl})$ & p Value & Mean difference $(95 \% \mathrm{Cl})$ & p Value \\
\hline LCQ total & $1.53(0.21$ to 2.85$)$ & $0.024^{*}$ & $0.01(-1.62$ to 1.64$)$ & 0.994 \\
\hline $\mathrm{CF}_{\text {per hour }}$ (fold change) & 0.59 (0.36 to 0.95$)$ & $0.030^{*}$ & 1.01 (0.55 to 1.86$)$ & 0.966 \\
\hline VAS severity & $-9.72(-20.80$ to 1.36$)$ & 0.084 & $1.6(-15.48$ to 18.74$)$ & 0.848 \\
\hline SF-36 PCS & $0.56(-2.52$ to 3.64$)$ & 0.717 & $0.48(-3.27$ to 3.37$)$ & 0.977 \\
\hline SF-36 MCS & $0.81(-3.10$ to 4.72$)$ & 0.680 & 0.72 (-3.06 to 4.51$)$ & 0.703 \\
\hline VPQ & $3.90(-0.33$ to 8.12$)$ & 0.070 & $-0.20(-3.43$ to 3.03$)$ & 0.901 \\
\hline HADS-Anxiety & $-0.42(-1.96$ to 1.13$)$ & 0.590 & $0.88(-0.57$ to 2.34$)$ & 0.225 \\
\hline HADS-Depression & $-0.44(-1.69$ to 0.81$)$ & 0.486 & $-0.18(-1.36$ to 0.99$)$ & 0.753 \\
\hline C2 (fold change) & $1.11(0.76$ to 1.61$)$ & 0.575 & NA & NA \\
\hline C5 (fold change) & $1.11(0.80$ to 1.54$)$ & 0.512 & NA & NA \\
\hline
\end{tabular}




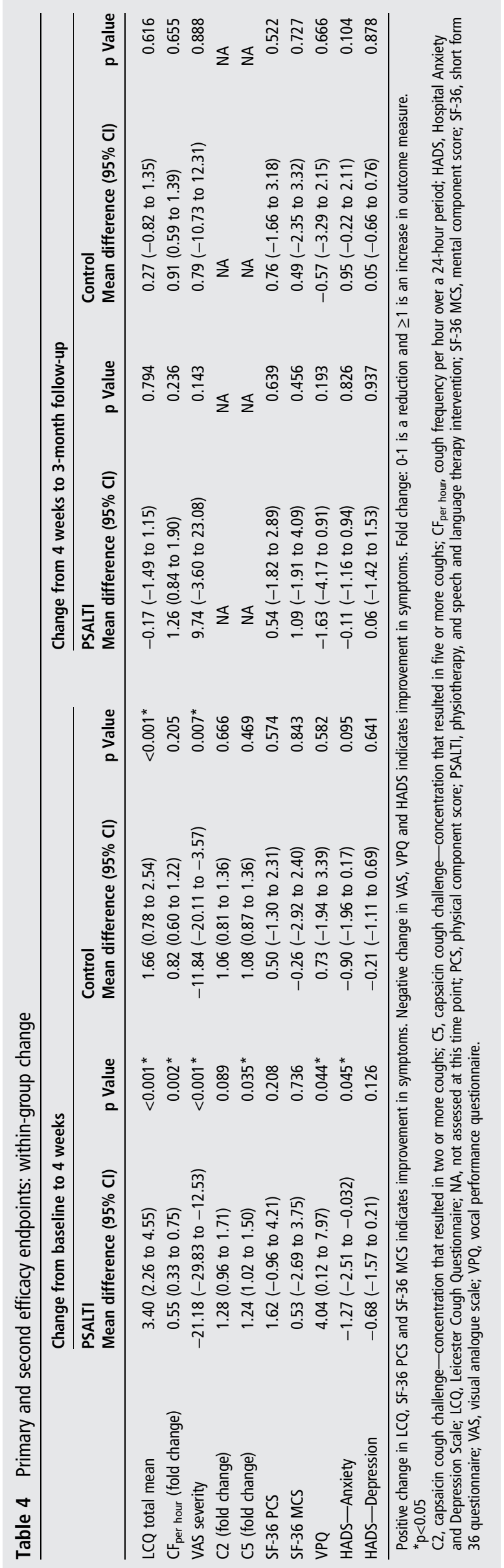

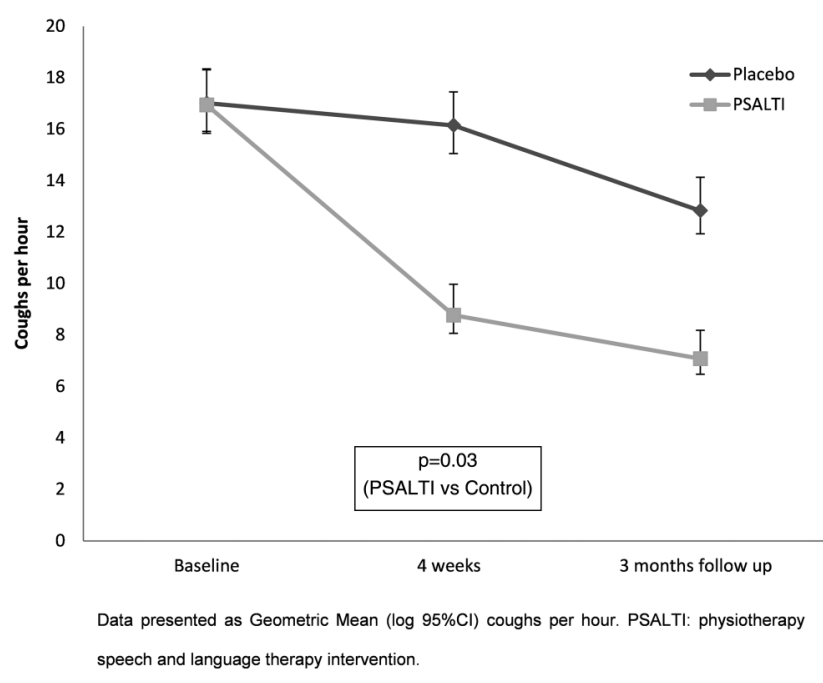

Figure 2 Change in objective cough frequency in physiotherapy, and speech and language therapy intervention (PSALTI) and control groups.

important outcome measure from the patient's perspective. ${ }^{7} 839$ The HRQoL of our participants was severely impaired, affecting physical, psychological and social domains, comparable with that reported in previous studies of refractory chronic cough. ${ }^{29} 40$ The improvement of HRQoL with PSALTI was large, LCQ 3.4 units. This improvement was greater than the MID of the LCQ, 1.3 units, ${ }^{27}$ and that reported for gabapentin therapy in patients with refractory chronic cough (LCQ improvement 2.5 units). ${ }^{12}$ The improvement with PSALTI was smaller when adjusted for the change in the control group (LCQ score, control group 1.66 units). HRQoL also improved with control intervention, but to a lesser extent than PSALTI. The aim of the control intervention was to provide participants with an equivalent quantity of clinical attention to the PSALTI intervention. This is additional to what most patients with refractory chronic cough would receive as usual care, since physiotherapy, and speech and language therapy services are not widely available for refractory chronic cough. It is possible that the control intervention had an antitussive effect and that the difference between PSALTI and control group may have been larger if compared with usual care (no active treatment). The control intervention was intended to be non-specific, but it is possible that some of its components such as stress/anxiety and lifestyle management may have had a positive benefit, particularly on the central sensitisation pathways that regulate cough.

The improvement in cough frequency assessed objectively with 24-hour cough monitoring supports the improvement in HRQoL with PSALTI occurred because of an actual reduction in coughing. Cough frequency outcome measures are increasingly being used as endpoints in clinical trials to validate the efficacy of antitussive therapy. ${ }^{41}{ }^{42}$ The LCM has been reported to be a valid method of counting coughs objectively. ${ }^{28}{ }^{43}$ An advantage of cough monitors over subjective measures is that they are not susceptible to the patient's or clinician's perception of cough severity. PSALTI was associated with an additional $41 \%$ reduction in cough frequency, which can be considered a large change, and is comparable with that observed with pharmacotherapy such as the P2X3 inhibitor AF-219. ${ }^{13}$ The minimal clinically important difference for cough monitor frequency in chronic cough has not been studied. The reduction of cough frequency was comparable with the minimal important difference reported for acute cough. ${ }^{42}$ We also assessed cough severity subjectively with VAS. 
There was a reduction in cough severity with PSALTI compared with control intervention, and the difference approached statistical significance. The reason for the discrepancy in effect size between HRQoL and VAS findings is not clear. A larger study would be needed to confirm whether PSALTI impacts cough severity assessed with VAS. Despite their widespread use, VAS have been poorly validated in comparison with HRQoL questionnaires and cough monitoring tools, as acknowledged by the American College of Chest Physicians' cough guidelines. ${ }^{31}$ There were no between-group differences in reported voice-related problems. We chose the VPQ, a patient-reported questionnaire, to assess voice, since it has been reported to have excellent internal consistency, repeatability and responsiveness. ${ }^{44}$ There are alternative questionnaires available to assess voice such as the voice handicap index and voice symptom scale. ${ }^{45}{ }^{46}$ A comparison of these scales by Webb $e a^{4} l^{44}$ concluded all were valid and reliable questionnaires for assessing patient's perceived voice dysfunction.

There were no adverse events associated with PSALTI, specifically no episodes of pulmonary infections. Patients with significant sputum production were excluded because of the potential risk of pulmonary infections associated with cough suppression. Longer term data with PSALTI are required to fully assess its safety. The mechanism by which PSALTI reduces cough is not clear, nor which component of PSALTI is most effective. PSALTI was not associated with a reduction in cough reflex sensitivity assessed with capsaicin when compared with the change in control group. There was however a significant within-group improvement in C5 in the PSALTI group, which indicates a reduction in cough reflex sensitivity, consistent with the studies by Ryan $e t a^{21}$ and Vertigan $e t a l,{ }^{15}$ who reported a reduction in cough reflex sensitivity with speech pathology management. The studies by Ryan $e t a l^{21}$ and Vertigan $e t a l^{15}$ however did not have a control group (no speech pathology management) for comparison. It is possible that we did not find a between-group difference in cough reflex sensitivity due to the small sample size of participants that underwent capsaicin cough challenge testing; further studies are needed to investigate this.

We investigated PSALTI in patients with refractory chronic cough. Our participants had a troublesome chronic cough despite numerous investigations and trials of therapy. A refractory chronic cough may also be referred to as idiopathic, difficult-to-treat, unexplained, sensory neuropathic and vagal neuropathy cough, although some differences exist between groups. ${ }^{47}$ PSALTI-type treatments have only been studied in patients with refractory chronic cough once they have undergone extensive investigations and/or trials of therapy. The role of PSALTI-type treatments earlier in the management of such patients has not been explored and needs to be studied. The efficacy of PSALTI is also unknown in other difficult-to-treat coughs, such as that associated with lung cancer, idiopathic pulmonary fibrosis and sarcoidosis, and this should be investigated. Further studies are needed to explore the optimum frequency and duration of PSALTI and other non-pharmacological treatments.

There were some limitations to our study. The study was single-blinded. It was not possible to blind the treating therapist to the intervention the participant received. The possibility that unconscious bias could have been conveyed to participants during the course of intervention cannot therefore be discounted. Double-blinding is not possible in studies of behavioural intervention. The potential bias was minimised by asking participants to complete their primary outcome measures independently from the treating therapist, and participants remained blinded until after completion of the final post-intervention outcome measures. Capsaicin cough reflex tests in some participants were performed by the treating therapist, but it is unlikely that this influenced the outcome, since our findings suggest no change with intervention when adjusted for control. Some components of PSALTI were tailored to the individual, according to clinical need. This may be considered both a limitation and strength, since it reduces the uniformity of intervention delivered, but reflects real-life clinical practice addressing the needs of an individual. Our study did not meet the intended sample size. This may have affected the power of our analyses and undermined the robustness of the results. Despite this, there was a clinically and statistically significant improvement in the primary outcome measure with PSALTI. Thirty-six (22\%) of the participants screened were uncontactable or declined to participate. The clinical characteristics of participants recruited were however consistent with the previously reported studies of refractory chronic cough. ${ }^{29} \mathrm{~A}$ significant number of participants were lost to follow-up for the 3-month follow up visit where secondary endpoints were assessed. This was largely from the control group. There was no significant difference in LCQ between groups at 3 months; this could be a consequence of a smaller sample size or a reduction in the long-term benefit of PSALTI following cessation of therapy. The long-term benefits of PSALTI need to be confirmed in larger studies. It is possible that some of the benefits of PSALTI may have been a consequence of intense supervision. The control intervention was however identical in frequency and duration of visits.

In conclusion, PSALTI is an effective therapy for patients with refractory chronic cough. It is associated with a significant improvement in health-related quality of life and cough frequency, compared with control. The optimal components of PSALTI and number of sessions of therapy need to be determined in future studies. The effectiveness of PSALTI used earlier in the treatment of chronic cough and other patient groups with difficult-to-treat cough should be evaluated. There is also a need for improved access to physiotherapy, and speech and language therapy services for patients with refractory chronic cough.

\section{Author affiliations}

${ }^{1}$ Division of Asthma, Allergy and Lung Biology, King's College London, London, UK

${ }^{2} S$ chool of Health and Rehabilitation, Keele University, Keele, UK

${ }^{3}$ King's College London, Denmark Hill Campus, London, UK

${ }^{4}$ Speech and Language Therapy Department, King's College Hospital, London, UK

${ }^{5}$ Department of Primary Care and Public Health Sciences, King's College London, London, UK

${ }^{6}$ NIHR Biomedical Centre, King's College London, London, UK

${ }^{7}$ Respiratory Medicine, Northumbria Healthcare NHSFT, North Tyneside General Hospital, North Shields, UK

${ }^{8}$ Centre for Respiratory Medicine and Allergy, Institute of Inflammation and Repair, The University of Manchester and Lancashire Teaching Hospitals NHS Foundation Trust, Preston, UK

${ }^{9}$ Speech and Language Therapy Department, Leighton Hospital, Mid Cheshire Hospitals Trust, Leighton, UK

${ }^{10} \mathrm{NIHR}$ Respiratory Biomedical Research Unit at the Royal Brompton NHS

Foundation Trust and Imperial College London, London, UK

${ }^{11}$ Physiotherapy Department, University College London Hospitals NHS Foundation Trust, London, UK

Acknowledgements This study represents independent research supported by the National Institute for Health Research (NIHR)/Wellcome Trust King's Clinical Research Facility and the NIHR Biomedical Research Centre and Dementia Unit at South London and Maudsley NHS Foundation Trust and King's College London. The views expressed are those of the authors and not necessarily those of the NHS, the NIHR or the Department of Health. All authors would like to acknowledge a number of key people who helped with this trial. The authors thank Dr Nicholas Hart for his help with applying for NIHR grant funding; Janet Mills and Maureen Armstrong for their help with recruitment of participants and study/data management at Royal Preston and North Tyneside Hospitals, respectively; Natasha Muzengi, Rachel Harding, Aliya Kaaba and Katie Pidgeon who all helped with conducting 
assessments and participant recruitment at King's College Hospital and Dr Jane Hutton, Consultant Clinical psychologist at King's College Hospital and the Institute of Psychiatry who helped to develop the psycho-educational counselling information booklet which was used as part of PSALTI.

Contributors Conception/design of work: RG, SSB, KKL and HB; Study recruitment: SAFCM, SSB, SMP, SJF, JHH and KFC; Assessments/treatment delivering in the trial: SAFCM, LC, JE and SL; Data analysis: SAFCM, AD, AP and SSB; Drafting manuscript: SAFCM, RG and SSB; Revised manuscript: All.

Funding This work was supported by a grant from the Chartered Society of Physiotherapy Charitable Trust, UK (Award PRF 10/4). Additional funding was obtained from NIHR-CRN. SSB was supported by King's College Hospital NHS Foundation Trust and London National Institute for Health Research (NIHR)/Wellcome Trust King's Clinical Research Facility and the NIHR Biomedical Research Centre and Dementia Unit at South London and Maudsley NHS Foundation Trust and King's College London. $A D$ acknowledges financial support from the National Institute for Health Research Biomedical Research Centre at Guy's and St Thomas' National Health Service (NHS) Foundation Trust and King's College London. AD also acknowledges support from the NIHR Collaboration for Leadership in Applied Health Research and Care South London (CLAHRC South London). The views expressed are those of the authors and not necessarily those of the NHS, the NIHR or the Department of Health.

Competing interests AP reports grants from NIHR, personal fees from Industry (Allergan, Merz and IPSEN), grants and non-financial support from Industry (Allergan and Biometrics), outside the submitted work. KFC has no disclosure for the work under consideration. Outside this work, he has received honoraria for participating in Advisory Board meetings regarding treatments for asthma and COPD for GSK, AZ, Novartis and J\&J, and has received grant funding through his institution from Pfizer, GSK and Merck.

Ethics approval London-Chelsea National Research Ethics Service (NRES) Committee.

Provenance and peer review Not commissioned; externally peer reviewed.

\section{REFERENCES}

1 Morice AH, McGarvey L, Pavord I, On behalf of the British Thoracic Society Cough Guideline Group. Recommendations for the management of cough in adults. Thorax 2006:61(Suppl 1):i1-24.

2 Ford AC, Forman D, Moayyedi P, et al. Cough in the community: a cross sectional survey and the relationship to gastrointestinal symptoms. Thorax 2006;61:975-9.

3 Morice A. Chronic cough: epidemiology. Chron Respir Dis 2008;5:43-7.

4 Morice AH, Fontana GA, Sovijarvi AR, et al. The diagnosis and management of chronic cough. Eur Respir J 2004;24:481-92.

5 Irwin R, Baumann MH, Bolser DC, et al. Diagnosis and management of cough executive summary: ACCP evidence-based clinical practice guidelines. Chest 2006;129(1 Suppl):1S-23S.

6 Haque RA, Usmani OS, Barnes PJ. Chronic idiopathic cough: a discrete clinical entity? Chest 2005;127:1710-13.

7 Brignall K, Jayaraman B, Birring SS. Quality of life and psychosocial aspects of cough. Lung 2008;186(Suppl 1):S55-8.

8 Birring SS, Prudon B, Carr AJ, et al. Development of a symptom specific health status measure for patients with chronic cough: Leicester Cough Questionnaire (LCQ). Thorax 2003;58:339-43.

9 French $\mathrm{CL}$, Irwin RS, Curley FJ, et al. Impact of chronic cough on quality of life Arch Intern Med 1998;158:1657-61.

10 Chung KF. Clinical cough VI: the need for new therapies for cough: disease-specific and symptom-related antitussives. Handb Exp Pharmacol 2009;187:343-68.

11 Belvisi MG, Geppetti P. Cough. 7: Current and future drugs for the treatment of chronic cough. Thorax 2004;59:438-40.

12 Ryan NM, Birring SS, Gibson PG. Gabapentin for refractory chronic cough: randomised, double-blind, placebo-controlled trial. Lancet 2012;380:1583-9.

13 Abdulqawi R, Dockry R, Holt K, et al. P2X3 receptor antagonist (AF-219) in refractory chronic cough: a randomised, double-blind, placebo-controlled phase 2 study. Lancet 2015;385:1198-205.

14 Morice AH, Menon MS, Mulrennan SA, et al. Opiate therapy in chronic cough. Am J Respir Crit Care Med 2007;175:312-15.

15 Vertigan AE, Kapela SL, Ryan NM, et al. Pregabalin and speech pathology combination therapy for refractory chronic cough: a randomized controlled trial. Chest 2016:149:639-48.

16 Jeyakumar A, Brickman TM, Haben M. Effectiveness of amitriptyline versus cough suppressants in the treatment of chronic cough resulting from postviral vagal neuropathy. Laryngoscope 2006;116:2108-12.
17 Chamberlain S, Birring SS, Garrod R. Nonpharmacological interventions for refractory chronic cough patients: systematic review. Lung 2014;192:75-85.

18 Chamberlain S, Garrod R, Birring SS. Cough suppression therapy: does it work? Pulm Pharmacol Ther 2013:26:524-7.

19 Vertigan AE, Theodoros DG, Gibson PG, et al. Efficacy of speech pathology management for chronic cough: a randomised placebo controlled trial of treatment efficacy. Thorax 2006;61:1065-9.

20 Patel AS, Watkin G, Willig B, et al. Improvement in health status following cough-suppression physiotherapy for patients with chronic cough. Chron Respir Dis 2011;8:253-8

21 Ryan NM, Vertigan AE, Bone $S$, et al. Cough reflex sensitivity improves with speech language pathology management of refractory chronic cough. Cough 2010;6:5

22 Ryan NM, Vertigan AE, Gibson PG. Chronic cough and laryngeal dysfunction improve with specific treatment of cough and paradoxical vocal fold movement. Cough 2009;5:4.

23 Vertigan A, Theodoros D, Winkworth A, et al. Chronic cough: a tutorial for speech language pathologists. J Med Speech Lang Pathol 2007;15:189-206.

24 Department of Health. Start active, stay active. UK: Department of Health, 2011.

25 NHS. Eight tips for healthy eating. 2013. http://www.nhs.uk/Livewell/Goodfood/ Pages/eight-tips-healthy-eating.aspx (accessed 05/10/2015)

26 NHS. Relaxation tips to relieve stress. 2013. http://www.nhs.uk/Conditions/ stress-anxiety-depression/Pages/ways-relieve-stress.aspx (accessed 05/10/2015).

27 Raj AA, Pavord DI, Birring SS. Clinical cough IV: what is the minimal important difference for the Leicester Cough Questionnaire? Handb Exp Pharmacol 2009; (187):311-20.

28 Birring SS, Fleming T, Matos S, et al. The Leicester Cough Monitor: preliminary validation of an automated cough detection system in chronic cough. Eur Respir J 2008:31:1013-18.

29 Yousaf $\mathrm{N}$, Monteiro W, Parker $\mathrm{D}$, et al. Long-term low-dose erythromycin in patients with unexplained chronic cough: a double-blind placebo controlled trial. Thorax 2010;65:1107-10.

30 Morice AH, Fontana GA, Belvisi MG, et al. ERS quidelines on the assessment of cough. Eur Respir J 2007;29:1256-76.

31 Boulet LP, Coeytaux RR, McCrory DC, et al. Tools for assessing outcomes in studies of chronic cough: CHEST guideline and expert panel report. Chest 2015:147:804-14.

32 Carding PN, Horsley IA. An evaluation study of voice therapy in non-organic dysphonia. Eur J Disord Commun 1992:27:137-58.

33 Vertigan $A E$, Theodoros DG, Winkworth AL, et al. Perceptual voice characteristics in chronic cough and paradoxical vocal fold movement. Folia Phoniatr Logop 2007:59:256-67.

34 Brazier JE, Harper R, Jones NM, et al. Validating the SF-36 health survey questionnaire: new outcome measure for primary care. BMJ 1992;305:160-4.

35 Zigmond AS, Snaith RP. The hospital anxiety and depression scale. Acta Psychiatr Scand 1983:67:361-70.

36 Lacson E, Xu J, Lin SF, et al. A comparison of SF-36 and SF-12 composite scores and subsequent hospitalization and mortality risks in long-term dialysis patients. Clin J Am Soc Nephrol 2010;5:252-60.

37 Birring SS, Pavord ID. Assessment of gender differences in health status with the Leicester Cough Questionnaire (LCQ). Thorax 2009;64:1008-9.

38 Little RJ, D'Agostino R, Cohen ML, et al. The prevention and treatment of missing data in clinical trials. N Engl J Med 2012;367:1355-60.

39 Spinou A, Birring SS. An update on measurement and monitoring of cough: what are the important study endpoints? J Thorac Dis 2014;6(Suppl 7):S728-34.

40 Birring SS, Murphy AC, Scullion JE, et al. Idiopathic chronic cough and organ-specific autoimmune diseases: a case-control study. Respir Med 2004;98:242-6

41 Lee KK, Savani A, Matos $\mathrm{S}$, et al. Four-hour cough frequency monitoring in chronic cough. Chest 2012;142:1237-43.

42 Lee KK, Matos S, Evans DH, et al. A longitudinal assessment of acute cough. Am J Respir Crit Care Med 2013:187:991-7.

43 Yousaf N, Monteiro W, Matos S, et al. Cough frequency in health and disease. Eur Respir / 2013:41:241-3.

44 Webb AL, Carding PN, Deary IJ, et al. Optimising outcome assessment of voice interventions, I: reliability and validity of three self-reported scales. J Laryngol Oto 2007;121:763-7.

45 Jacobson $\mathrm{BH}$, Johnson A, Grywalski C, et al. The voice handicap index (VHI): development and validation. Am I Speech Lang Pathol 1997;6:66-70.

46 Deary IJ, Wilson JA, Carding PN, et al. VoisS: a patient-derived Voice Symptom Scale. J PsychoSom Res 2003;54:483-9.

47 Birring SS. Controversies in the evaluation and management of chronic cough. Am J Respir Crit Care Med 2011;183:708-15. 